\title{
Effectual Approach for Facial Expression Recognition System
}

\author{
Shalini Mahto ${ }^{1}$, Mrs. Yojana Yadav ${ }^{2}$ \\ ME Scholar, Department of ET \&T, Chhatrapati Shivaji Institute of Technology, Durg, India ${ }^{1}$ \\ Associate Professor, Department of ET \&T, Chhatrapati Shivaji Institute of Technology, Durg, India ${ }^{2}$
}

\begin{abstract}
Automatic facial expression is an interesting and challenging problem, and impacts important applications in many areas such as human-computer interaction and data-driven animation. Facial expression recognition is the process performed by computers which consist of detect the face in the image, and pre-process the face regions, extracting facial expression features from image by analysing the change in the appearance of facial features and then classifying this information into facial expression categories like fear, happy sad etc. In this research work, an Automatic Facial Expressions Recognition System is presented that recognizes five principal expressions that are Happy, Sad, Neutral, Anger and Disgust. The system uses an efficient approach for the recognition of those expressions on the basis of some extracted features. The whole system is implemented on the dataset of 150 images of frontal facial expressions of happy, sad, neutral, anger and disgust by using MATLAB. The images are collected from the Karolinska Directed Emotional Faces (KDEF) database. We empirically evaluate the facial representation based on local binary pattern (LBP) features. Then recognition performed by KNN classifier with LBP features. The result obtained after implementation is very good.
\end{abstract}

Keywords: Face Detection, Viola Jones, Feature Extraction, LBP (Local Binary Pattern), KNN Classifier.

\section{INTRODUCTION}

Expressions are the most powerful and simplest way to expression recognition is shown in Fig. 1. Preprocessing express feelings and emotions between human beings. It is part includes four functions auto color, auto brightness, a medium of communication for describing any situation. and auto contrast. In facial feature extraction step face Human communication have two main types one of it is detection, segmentation by edge detection and feature verbal that is auditory and the second one is non-verbal extraction are performed. At last, based on the extracted that is visible. Facial expressions come into the non-verbal features and proposed approach the expressions will be type of communication. Facial expression recognition solves the problem of face detection, facial feature extraction and expression recognition. Because of these, the facial expression recognition becomes the most interested and active research topic into last two decades. Some of the common application areas related to face and facial expressions is video conferencing, face identification and verification, security, mobile applications and human computer interactions. Mostly, Facial Expression Recognition System have three key components face detection, facial feature extraction and expressions recognition. Face detection is the primary need of expression recognition system. Facial feature extraction includes the detection of nose, eyes and mouth parts from the face as facial information.

This paper proposes a fast and efficient approach for facial expression recognition that recognizes five principle expressions happy, sad, neutral, anger and disgust. The recognition system follows a procedure that includes preprocessing, face detection, feature extraction and expression recognition to recognize and classify the expressions.

\section{Methodology}

The Facial Expression Recognition System divides the task of expression recognition into three major parts; preprocessing, facial feature extraction and expressions classification. The step by step process for facial

classify into happy, sad and neutral expressions.

\section{A. Pre-processing}

Preprocessing is the most important and the required step of the image processing. It is performed to get uniform and noise free image for further processing. This step includes the following functions:

Auto Brightness- Auto brightness function adjust the brightness of the image.

Auto Contrast- Auto contrast function automatically calculates the favorable contrast for the image will increase the brightness of the image.

Auto Color- Auto color function adjust the color of the image.

\section{B. Facial Feature Extraction}

Facial feature extraction is the second foremost part of the facial expression recognition system. This comprises three phases: face boundary detection, detection and cropping of ROI (region of interest), and feature extraction. Face boundary detection phase is performed to identify the face in the image that contains eyes, nose and mouth. After that, segmentation is performed to identify the region of interest. In this research work eyes and mouth are taken as region of interest for the processing. 


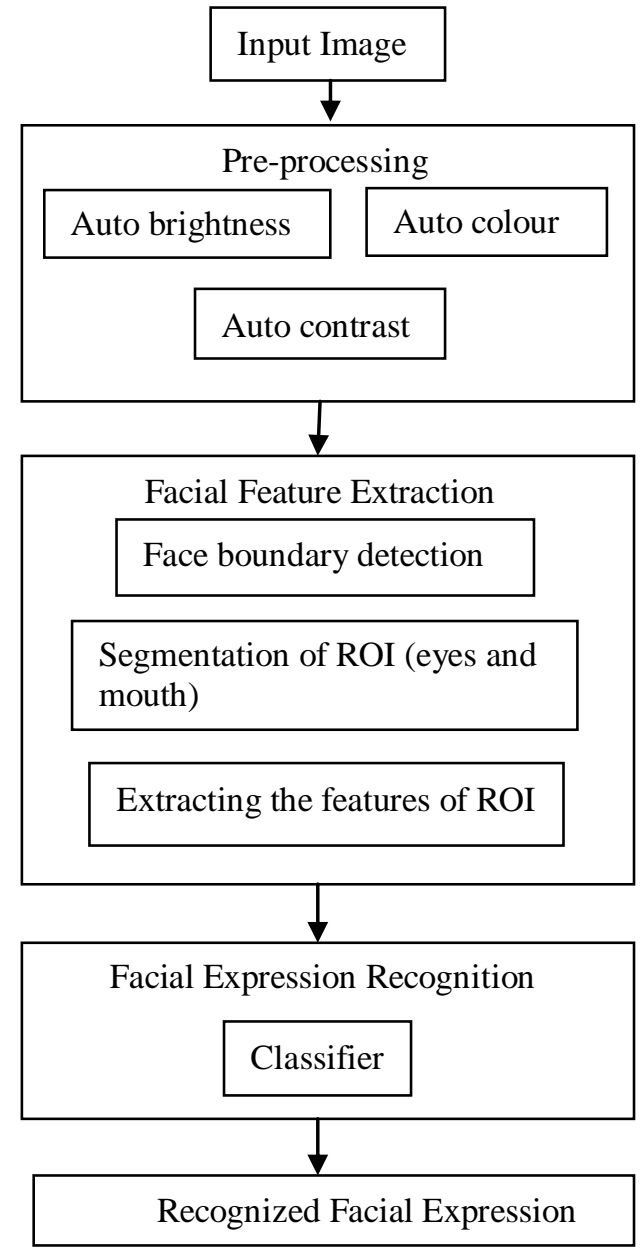

Fig. 1 Flow process of proposed Facial Expression Recognition System

1) Face Boundary Detection: Face boundary detection phase is also a very important step for the facial expression recognition. In this phase, the face boundary is detected and for that Viola Jones Method is used. Viola Jones method is a widely used method for face detection, its training is slow but detection is extremely fast and efficient. It is scale and location invariant detector. Fig. 2 is the detected face boundary from images of face.

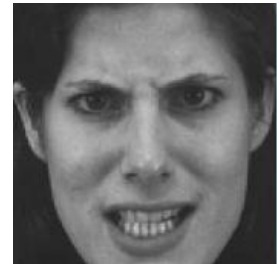

Fig. 2 Detected face boundary from image

2) Segmentation of ROI: Segmentation of image means partitioning the image into multiple parts. In this system segmentation is used to detect the interested regions such as eyes and mouth from images and for that also Viola Jones method is adopted. Face boundary detection and segmentation of ROI collectively the face detection process performed by the adopted method (Viola Jones). Fig. 2 represents the partitioning of original face for cropping ROI. 
International Journal of Advanced Research in Computer and Communication Engineering Vol. 4, Issue 3, March 2015

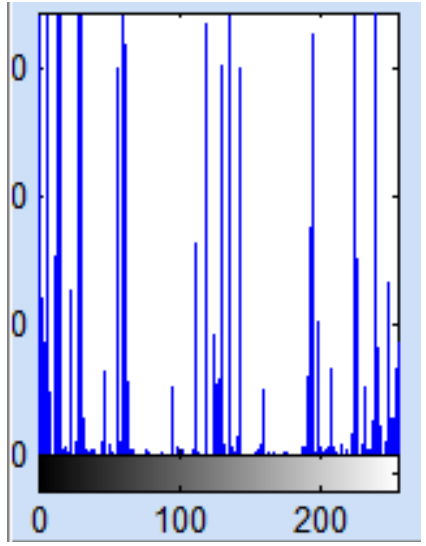

Fig. 5 Histogram of LBP coded eyes area

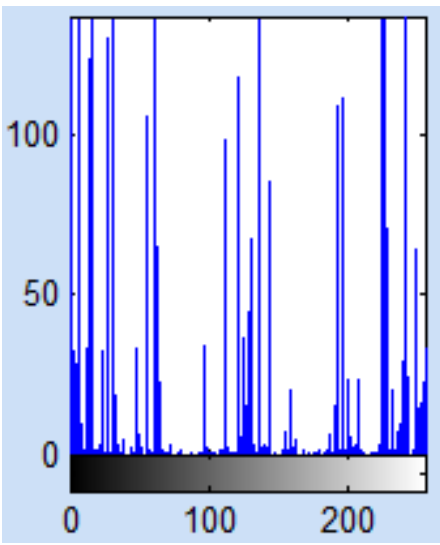

Fig. 6 Histogram of LBP coded mouth area

\section{Facial Expression Recognition:}

Facial Expression Recognition is the final step for Recognition System. This module uses KNN (K Nearest Neighbour) classifier. The K Nearest Neighbour algorithm is a non-parametric method used for classification and regression. The input consists of $\mathrm{K}$ closest training examples in the feature space. The output is a class membership. An object is classified by a majority vote of its neighbours, with the object being assigned to the class most common among its $\mathrm{k}$ nearest neighbours $(\mathrm{k}$ is a positive integer).

\section{III.EXPERIMENTAL RESULT}

In this research work, an Automatic Facial Expression Recognition System is presented that recognizes five principle expressions. The approach used to recognize the expressions is fast and efficient. Table I contains the recognition rate of the system that shows true recognition of all the expressions. A dataset of 30 images for each expression that is 150 images is collected. Images are taken from Karolinska Directed Emotional Faces (KDEF) database. The whole system is implemented using MATLAB and gives the average recognition rate of $96 \%$. Fig. 7 shows the success rate of the system. The proposed system interface is shown in fig. 8, which consists functions performed during the facial expression recognition.
TABLE I

RECOGNITION RATES OF EXPRESSIONS ON THE DATASET

\begin{tabular}{|l|l|l|l|}
\hline S. No. & Expressions & $\begin{array}{l}\text { Correct } \\
\text { Classification }\end{array}$ & $\begin{array}{l}\text { Classificatio } \\
\text { n Accuracy }\end{array}$ \\
\hline 1. & Anger & $28 / 30$ & $93.33 \%$ \\
\hline 2. & Disgust & $29 / 30$ & $96.66 \%$ \\
\hline 3. & Happy & $29 / 30$ & $96.66 \%$ \\
\hline 4. & Neutral & $30 / 30$ & $100 \%$ \\
\hline 5. & Sad & $28 / 30$ & $93.33 \%$ \\
\hline Total Images System & $144 / 150$ \\
\hline \multicolumn{2}{|l|}{$\begin{array}{l}\text { Whole } \\
\text { performance (\%) }\end{array}$} & $96 \%$ \\
\hline
\end{tabular}

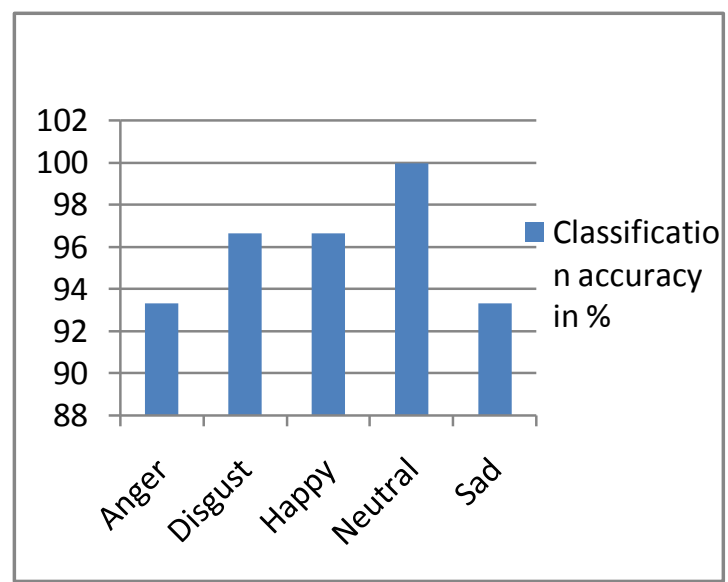

Fig. 7 The Success Rate of the System for the Recognition of five Expressions

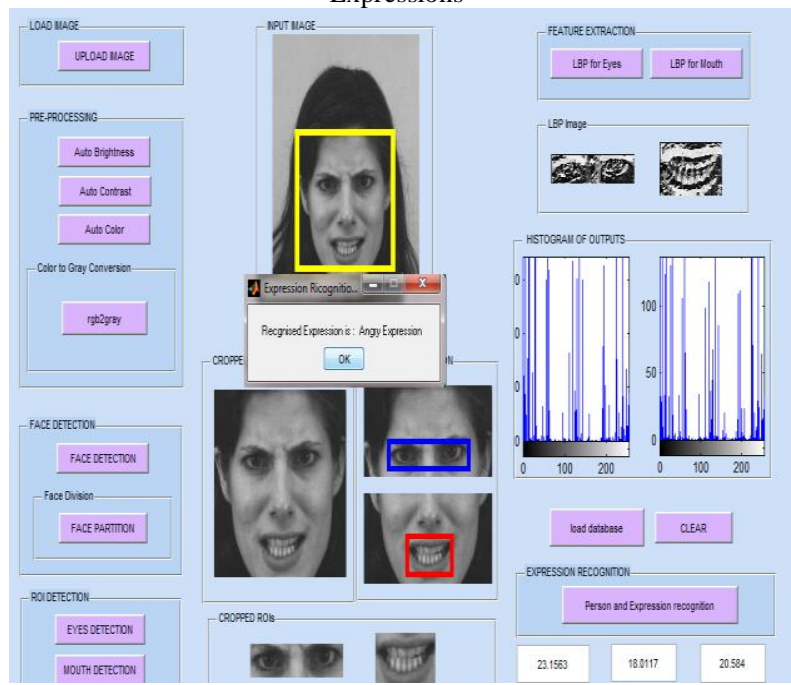

Fig. 8 Proposed Facial Expression Recognition System Interface

\section{IV.CONCLUSION}

In this paper, a fast and effective approach for facial expression recognition is presented that recognizes the five principal expressions happy, sad, neutral, disgust and anger. This new approach gives excellent and effective result. The system is implemented on the KDEF database that has frontal facial expression color images. After implementation and based on the experimental result it is observed that, the approach provides very good recognition rate and also effective for the frontal facial 
expression recognition. As for the future work, new expressions can be recognized with other interested regions such as eyes and nose. Also other techniques can be used for the recognition.

\section{REFERENCES}

[1] Caifeng Shan, Shaogang Gong, Peter W. McOwam, "Robust facial expression recognition using local binary patterns", IEEE international conference on image processing 2005 ICIP volume 2.

[2] C. P. Sumathi, T. Santhanam, and M. Mahadevi, "Automatic Facial Expression Analysis: A Survey", International Journal of Computer Science and Engineering Survey, (IJCSES), vol 3, no. 6, December 2012.

[3] Pushpaja V. Saudagare and D.S. Chaudhari, "Facial Expression Recognition using Neural Network- An Overview", International Journal of Soft Computing and Engineering (IJSCE), Vol. 2, Issue1, pp. 224-227, March 2012.

[4] Le Hoang Thai, Nguyan Do Thai Nguyan and Tran Son Hai, "A Facial Expression Classification System Integrating Canny, Principle Component Analysis and Artificial Neural Network", International Journal of Machine Learning and Computing, vol 1, no. 4, October 2011.

[5] Sachin D. More and Sachin Deshpande, "Fuzzy Model for Human Face Expression Recognition", International Journal of Advanced Technology and Engineering Research (IJATER), vol. 2, Issue 2, pp. 149-153, May 2012.

[6] Renuka R. Londe, Vrushshen P. Pawar, "Analysis of Facial Expression using LBP and Neural Network", International Journal of Computer Application (0975-8887), vol 44, no.21, April 2012.

[7] Mandeep Kaur and Rajeev Vashisht, "Comparative Study of Facial Expression Recognition Techniques", International Journal of Computer Applications (0975-8887), vol. 13, no. 1, pp. 43-50, January 2011

8] Amir Jamshidnezhad, Md Jan Nordin, "A Classifier Model based on the Features Quantitative Analysis for Facial Expression Recognition", International Conference on Advanced Science, Engineering and Information Technology, Jan 2011.

[9] Roja Ghasemi, Maryam Ahmadi, "Facial Expression Recognition Using Facial Effective Areas And Fuzzy Logic", Iranian Conference on Intelegent System (ICIS),2014.

[10] Anita C., M. K. Venkatesha and B. Suryanarayana Adiga, "A Survey on Facial Expression Databases", International Journal of Engineering and Technology, vol. 2, no. 10, pp. 5158-5174, 2010.

[11] Mohammad Javed, Bhaskar Gupta, "Performance Comparition ofVarious Face Detection Techniques", International Journal of Scientific Research Engineering and Technology (IJSRET), Volume 2 Issue 1, pp 019-0027, April 2013.

[12] Divyarajsinh N. Parmar, Brijesh B. Mehta, “ Face Recognition Methods \& Applications", International Journal of Computer Technology and Applications (IJCTA), Vol 4(1), 84-86.

\section{BIOGRAPHIES}

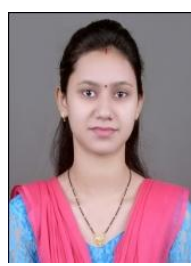

Shalini Mahto (B.E.) has completed her engineering in Electronics and Telecommunication Branch from Bhilai Institute of Technology, Durg, Chhattisgarh, India. She is pursuing M.E. in Communication Specialization from Chhatrapati Shivaji Institute of Technology, Durg, Chhattisgarh. She has published one research paper in international conference and one research paper in international journal. Her area of interest is image processing and data mining.

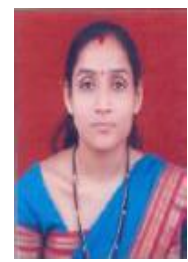

Mrs. Yojana Yadav (M. Tech) is currently working as associate professor in Electronics and Telecommunication Engineering in Chhatrapati Shivaji Institute of Technology, Durg, Chhattisgarh, India. She has 9 years experience of teaching. She has published six research papers in national/ international conferences and six research papers in international journals. Her area of interest is neural network and communication technology. 